\title{
FRECUENCIA DE HIDATIDOSIS EN NIÑOS Y ADOLESCENTES HOSPITALIZADOS EN EL INSTITUTO NACIONAL DE SALUD DEL NIÑO (PERIODO 1996-2005)
}

\author{
Frequency of Hydatidosis of Children and Adolescents Hospitalized \\ in the National Child Health Institute (Period 1996-2005)
}

Iván Huamán G. ${ }^{1}$, Luis Marocho C. ${ }^{2}$, Teresa López U. ${ }^{3}$, César Gavidia C. 1,4

\section{RESUMEN}

\begin{abstract}
El objetivo del presente estudio fue determinar la frecuencia de pacientes con hidatidosis en el Instituto Nacional de Salud del Niño (INSN), Lima, durante el periodo 1996-2005. Se revisaron 177 historias clínicas de un total de 235 pacientes con diagnóstico de hidatidosis. La frecuencia de pacientes hospitalizados con diagnóstico de hidatidosis fue de $0.21 \%$ (235/109 550). El mayor número de pacientes procedió del departamento de Lima $(33.9 \%$, 60/177), seguido por Junín y Pasco. El 55.4\% (98/177) de los pacientes fueron varones y el $50.8 \%$ (90/177) de los casos se dio en pacientes entre 5 y 9 años de edad. El 44.0\% (78/177) de pacientes sufrió de hidatidosis pulmonar y el 23.2\% (41/177) padeció de hidatidosis hepática. Los resultados indican que podría estar ocurriendo un ciclo urbano de la enfermedad.
\end{abstract}

Palabras clave: zoonosis, hidatidosis, Echinococcus granulosus, hombre, perro, Instituto Nacional de Salud del Niño, INSN

\section{Abstract}

The objective of the study was to determine the frequency of patients with hydatidosis at the Instituto Nacional de Salud del Niño (INSN), Lima, during the period 1996-2005. Clinical records of 177 patients out of 235 patients diagnosed withhydatidosis were analyzed. The frequency of patients with hydatidosis was $0.21 \%(235 / 109550)$. The largest number of patients were from Lima department $(33.9 \%, 60 / 177)$, followed by Junín and Pasco. The 55.4\% (98/177) of patients was male and 50.8\% (90/177) of patients were between 5 and 9 years of age. The $44.0 \%$ (78/177) of patients suffered pulmonary hydatidosis and $23.2 \%(41 / 177)$ had hepatic hydatidosis. Results indicated that an urban cycle of the diseases would be occurring.

Key words: zoonoses, hydatidosis, Echinococcus granulosus, man, dog, National Institute of Child Health, INSN

\footnotetext{
${ }^{1}$ Laboratorio de Medicina Veterinaria Preventiva, ${ }^{3}$ Laboratorio de Microbiología y Parasitología Veterinaria, Facultad de Medicina Veterinaria, Universidad Nacional Mayor de San Marcos, Lima

${ }^{2}$ Oficina de Epidemiología, Instituto Nacional de Salud del Niño, Lima

${ }^{4}$ E-mail: cmgavidia@yahoo.com
} 


\section{INTRODUCCIÓN}

La hidatidosis es una zoonosis parasitaria producida por la fase larvaria de un céstodo de la familia Taenidae, donde el Echinococcus granulosus es la especie de mayor difusión. La hidatidosis es endémica en los países de América del sur, principalmente en Argentina, sur de Brasil, Uruguay, Chile y Perú (Chuquisana et al., 2000). Se presenta mayormente en zonas agrícolas y ganaderas, donde se dan las condiciones necesarias para la presentación y propagación de esta enfermedad (Núñez et al., 2003).

El E. granulosus tiene como hospedador definitivo al perro y a otros cánidos silvestres y como hospedador intermediario a los ovinos, bovinos, porcinos, camélidos $\mathrm{y}$, accidentalmente, al hombre, afectando principalmente el hígado y los pulmones (Muñoz, 2007). El hospedador definitivo se infecta con el parásito al consumir vísceras crudas de hospedadores intermediarios que contienen estadios embrionarios (McManus et al., 2003).

De acuerdo con las estadísticas del Ministerio de Salud del año 2000, los departamentos de Cerro de Pasco, Huancavelica, Junín, Arequipa, Puno y Cusco registraron las tasas más altas de infección, con una máxima incidencia de 64.4 por cada 100000 habitantes (Stiglich et al., 2004). La hidatidosis es endémica en Cerro de Pasco, con una incidencia anual de $43 \times 10^{5}$ habitantes en 1993, incrementándose a $78.6 \times 10^{5}$ habitantes en el 2000. En la SAIS Túpac Amaru, empresa ganadera ubicada en el departamento de Junín, se registró una prevalencia de $9.1 \%$ (Moro et al., 1997).

En los últimos años se han reportado casos autóctonos en áreas urbanas no endémicas como en la ciudad de Lima y el departamento costero de Ica (Cabrera et al., 2005). Un estudio de seguimiento de pacientes con antecedentes de hidatidosis pulmonar en el Hospital Nacional Hipólito Unanue de
Lima, entre 1980 y 1986, reportó 41 casos autóctonos pertenecientes a Lima Metropolitana (Alarcón et al., 1992), en tanto que, en un estudio posterior (1990-1996) se encontró 51 casos (Huamán, 1999).

El hombre, por ignorancia, favorece el contacto entre el hospedador definitivo del parásito (perro) y los hospedadores intermediarios. La compleja naturaleza y el desconocimiento del problema por el hombre facilita la infección de los animales y de sí mismo, así como favorece la creación de condiciones ecológicas favorables al desarrollo de las formas larvarias (Sapunar, 1997). En el hombre, las manifestaciones clínicas se manifiestan, generalmente, en plena edad productiva, es decir, entre 18 y 30 años, provocando pérdidas económicas, no solo por el costo del diagnóstico, tratamiento y convalescencia, sino también por la merma en el rendimiento laboral.

Datos referentes a la casuística y las variables que posibilitan el estudio epidemiológico en pacientes con hidatidosis son limitados en el país. Por otro lado, los estudios realizados en los hospitales de la capital fueron en pacientes adultos, pese a que, en la mayoría de los casos, la infección afecta a los niños (Noemí et al., 2003). En base a esto, se diseñó el presente estudio a fin de determinar la frecuencia de pacientes pediátricos con diagnóstico de hidatidosis entre los años 1996-2005 para conocer la dinámica de la enfermedad.

\section{Materiales y Métodos}

Se realizó un estudio retrospectivo de tipo descriptivo mediante una revisión de historias clínicas de los pacientes dados de alta con diagnóstico de hidatidosis, que fueron atendidos en los servicios del Instituto Nacional de Salud del Niño (INSN), Lima, entre enero de 1996 y diciembre de 2005. No obstante, no se pudo contar con algunas fichas, debido que por política institucional, los regis- 
Cuadro 1. Egresos hospitalarios con diagnóstico de hidatidosis, por grupo de edad y género, en el Instituto Nacional de Salud del Niño (INSN), Lima - Perú. 1996-2005

\begin{tabular}{lcccc}
\hline \multirow{2}{*}{$\begin{array}{l}\text { Edad } \\
\text { (años) }\end{array}$} & N. & $\begin{array}{c}\text { Masculino } \\
(\%)\end{array}$ & $\begin{array}{c}\text { Femenino } \\
(\%)\end{array}$ & $\begin{array}{c}\text { Total } \\
(\%)\end{array}$ \\
\hline 1 a 4 & 18 & 6.1 & 15.2 & 10.2 \\
5 a 9 & 90 & 52.0 & 49.4 & 50.8 \\
10 a 14 & 59 & 36.7 & 29.1 & 33.3 \\
>15 & 10 & 5.1 & 6.3 & 5.6 \\
\hline Total & 177 & 55.4 & 44.6 & 100 \\
\hline
\end{tabular}

Cuadro 2. Egresos hospitalarios según localización del quiste hidatídico en el Instituto Nacional de Salud del Niño (INSN), Lima - Perú. 1996-2005

\begin{tabular}{lcc}
\hline Diagnóstico de hidatidosis & N. ${ }^{\circ}$ & $\%$ \\
\hline Pulmonar y hepática & 45 & 25.4 \\
Pulmonar derecha complicada & 39 & 22.0 \\
Hepática & 28 & 15.8 \\
Pulmonar izquierda complicada & 17 & 9.6 \\
Hepática complicada & 13 & 7.3 \\
Pulmonar bilateral & 10 & 5.7 \\
Otras localizaciones & 7 & 4.0 \\
Pulmonar izquierda & 5 & 2.8 \\
Pulmonar derecha & 4 & 2.3 \\
Pulmonar bilateral complicada & 3 & 1.7 \\
Pulmonar y otras localizaciones & 2 & 1.1 \\
Pulmonar bilateral y hepática & 2 & 1.1 \\
Hepática y otras localizaciones & 2 & 1.1 \\
\hline Total & 177 & 100 \\
\hline
\end{tabular}

tros se eliminan cuando un paciente cumple 18 años o deja de atenderse en el hospital por cuatro años. Además, tampoco se tomó en consideración las historias clínicas de pacientes atendidos por consulta externa por carecer de la información necesaria para el análisis.
Se colectó datos referentes al género, edad, año, procedencia, tiempo de hospitalización, diagnóstico definitivo, tipo y resultado de tratamiento, contacto con hospederos definitivos e intermediarios, antecedentes de hidatidosis familiar, ocupación familiar, dis- 
Cuadro 3. Egresos hospitalarios con diagnóstico de hidatidosis, según tiempo de hospitalización en el Instituto Nacional de Salud del Niño (INSN), Lima - Perú. 1996-2005

\begin{tabular}{ccc}
\hline Días de hospitalización & N. ${ }^{\circ}$ de casos & $\%$ \\
\hline 1 a 20 & 73 & 41.2 \\
21 a 40 & 53 & 29.9 \\
41 a 60 & 25 & 14.1 \\
>60 & 26 & 14.7 \\
\hline Total & 177 & 100 \\
\hline
\end{tabular}

Cuadro 4. Síntomas de pacientes con diagnóstico de hidatidosis en el Instituto Nacional de Salud del Niño (INSN), Lima - Perú. 1996-2005 ( $\mathrm{n}=177$ )

\begin{tabular}{lcc}
\hline \multirow{2}{*}{ Síntomas } & \multicolumn{2}{c}{ Casos } \\
\cline { 2 - 3 } & N. & $\%$ \\
\hline Tos & 100 & 56.5 \\
Fiebre & 65 & 36.7 \\
Hemoptisis & 60 & 33.9 \\
Dolor abdominal & 49 & 27.7 \\
Disnea & 44 & 24.9 \\
Dolor torácico & 43 & 24.3 \\
Vómito & 42 & 23.7 \\
Tumoración (hipocondrio derecho) & 38 & 21.5 \\
Ictericia & 10 & 5.6 \\
\hline
\end{tabular}

ponibilidad de servicios básicos, condición socioeconómica, y resultados del diagnóstico por examen clínico, tanto por imágenes como por serología.

La frecuencia de niños y adolescentes con hidatidosis se calculó en base a los casos positivos y el total de pacientes hospitalizados. La elaboración de distribuciones de frecuencia, en relación a las variables en estudio, sehizo con el programa estadístico SPSS 9.0.

\section{Resultados}

El número total de pacientes hospitalizados con diagnóstico de hidatidosis en el INSN en el periodo de estudio fue de 235 casos (Fig. 1), pero solo se obtuvo la historia clínica de 177 pacientes. El número total de pacientes fue de 109550 en el mismo periodo, lo que da una frecuencia de pacientes con hidatidosis de $0.21 \%$, que equivale a 215 casos X $10^{5}$ egresos hospitalarios. 


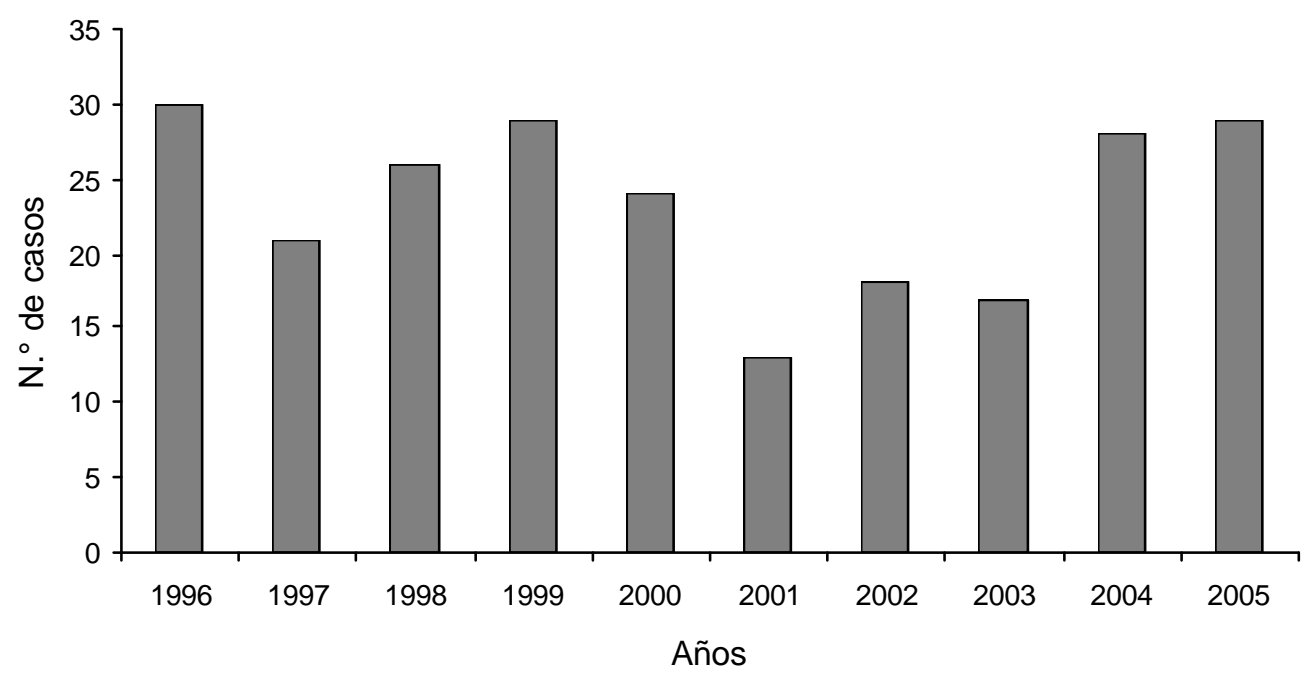

Figura 1. Egresos hospitalarios con diagnóstico de hidatidosis en el Instituto Nacional de Salud del Niño (INSN), Lima - Perú. 1996-2005

El 55.4\% (98/177) de los pacientes con hidatidosis fue de sexo masculino y el $50.8 \%$ (90/177) de los casos se observó en pacientes entre 5 y 9 años de edad (Cuadro 1). El $73.5 \%$ (130/177) de los pacientes procedían de los departamentos de Lima, Junín y Cerro de Pasco, siendo Lima el de mayor número de casos $(33.9 \%, 60 / 177)$. Dentro de la ciudad de Lima, los pacientes provinieron principalmente de los distritos de Comas, El Agustino, San Juan de Lurigancho y Ate Vitarte $(30.2 \%, 19 / 60)$.

El tipo y frecuencia de hidatidosis diagnosticados se muestra en el Cuadro 2. Se observa que el $44.0 \%$ (78/177) de pacientes sufrió de hidatidosis pulmonar, el 23.2\% (41/ 177) padeció de hidatidosis hepática, el $26.6 \%$ (47/177) de hidatidosis pulmonar y hepática, y el $6.2(11 / 177)$ de hidatidosis localizada en otros órganos.

El tiempo de hospitalización más frecuente fue entre 1 y 20 días $(41.2 \%, 73 / 177)$ (Cuadro 3), y el síntoma más común fue la tos $(56.5 \%, 100 / 177$; Cuadro 4). La resolución de la patología fue quirúrgica en el 93.8\% de los casos.

\section{Discusión}

La frecuencia de pacientes con hidatidosis entre 1996-2005 (0.215\%) fue similar a la frecuencia hallada entre 1990-1995 (0.235\%, Dávila, 1996). Posiblemente, uno de los factores importantes que no permitiría disminuir la frecuencia de casos de hidatidosis es la falta de un programa de control o el desconocimiento y falta de aplicación de este en el país. En los Andes centrales, áreas consideradas endémicas para la hidatidosis, el programa piloto de control no ha estado operativo desde 1980; este programa logró disminuir la prevalencia de la infección por Echinococcus en perros y ovejas a niveles de 1.6 y $5 \%$, respectivamente, antes de ser desmantelado (Moro et al., 1997).

La mayor ocurrencia de casos entre 5 y 9 años de edad (50.8\%) concuerda con un estudio sobre hidatidosis pulmonar pediátrica en el Hospital Cayetano Heredia, donde se encontró $64 \%$ de casos en niños menores de 10 años (Stiglich et al., 2004). Esto indica que el contacto con el agente causal ocurre en edades muy tempranas, ya que el creci- 
miento del quiste es de, aproximadamente, 1 cm por año (Noemí et al., 2003). Los niños suelen ser más descuidados en los hábitos de higiene y establecen relaciones más estrechas con sus mascotas y, por lo tanto, son el grupo etáreo más susceptible a infectarse con E. granulosus (Tamayo et al., 2004).

La mayoría de los casos provinieron del departamento de Lima, en parte debido a la gran migración de pobladores de la Sierra, ya que traen sus costumbres, formas de vida y animales posiblemente infectados. En los departamentos de la sierra central del Perú, el $E$. granulosus es considerado endémico debido a la crianza de ovejas y, principalmente, a la falta de educación sanitaria (Moro et al., 1997). La incidencia anual en el departamento de Pasco para el año 2000 fue de 78.6 x 100000 habitantes (Núñez et al., 2003).

Los habitantes de los distritos de Lima de donde provinieron la mayoría de casos (Comas, El Agustino, Ate Vitarte y San Juan de Lurigancho) se compone mayormente de población migrante. Es posible que esté ocurriendo un ciclo urbano de transmisión de la hidatidosis dado que la mayoría de los pacientes con hidatidosis no indican haber retornado hacia zonas endémicas, y esto podría facilitarse por la existencia de mataderos clandestinos y la falta de educación sanitaria, tal como ocurrió en la ciudad costera de Chincha, donde se demostró un foco endémico de equinococosis humana y canina (Moro et al., 2004).

La hidatidosis pulmonar fue el tipo de mayor frecuencia (44.0\%), y similar a reportes en la población infantil de Chile (Retamal et al., 1994), y esto es probablemente debido al tipo de parénquima que puede distenderse con facilidad permitiendo el crecimiento del quiste hidático (Jerray et al., 1992). Los datos referidos a pacientes adultos demuestran que la hidatidosis hepática es la forma más frecuente de presentación de esta enfermedad (Lozano et al., 2004).
El tiempo de estancia hospitalaria es variable, dependiendo de factores tales como localización del quiste, complicaciones y estado general del paciente. En el presente estudio se encontró que el $41.2 \%$ de pacientes tuvo un tiempo de hospitalización entre 1 y 20 días, tiempo relativamente corto si se considera que la resolución de la patología fue quirúrgica en la mayoría de los casos (93.8\%). Este periodo fue menor en comparación con los 31 días de promedio de estancia hospitalaria en el Hospital Cayetano Heredia (Stiglich et al., 2004). Muchos pacientes del INSN son derivados de hospitales del interior del país y llegan con diagnóstico de hidatidosis complicada para ser intervenidos quirúrgicamente, lo cual puede explicar la menor estancia hospitalaria.

\section{Conclusiones}

? La frecuencia de egresos hospitalarios con diagnóstico de hidatidosis en el Instituto Nacional de Salud del Niño (INSN), Lima, entre 1996 y 2005, fue de 215 x 100000 egresos hospitalarios.

? $\quad$ El 73.5\% (130/177) de pacientes hospitalizados con diagnóstico de hidatidosis provinieron de los departamentos de Lima, Junín y Cerro de Pasco.

? La hidatidosis pulmonar fue el tipo de hidatidosis que se presentó en mayor cantidad de pacientes $(44.0 \%)$, seguida de la hidatidosis hepática (23.2\%) y en menor proporción en otras localizaciones.

\section{Literatura Citada}

1. Alarcón J, Somocurcio J, Piscoya J, Reyes $N$, Arévalo $N$, Bustamante $E$. 1992. Hidatidosis pulmonar: Estudio epidemiológico de casos urbanos en el Hospital Hipólito Unanue de Lima. Rev Per Epidemiol 5(2): 15-19. 
2. Cabrera R, Talavera E, TrilloAltamirano M. 2005. Conocimientos, actitudes y prácticas de los matarifes acerca de la hidatidosis/equinococosis, en dos zonas urbanas del departamento de Ica, Perú. An Fac Med, Perú 66(3): 203-211.

3. Chuquisana J, Chávez A, Casas E. 2000. Determinación de Echinococcus granulosus en perros del cono Norte de Lima. Rev Inv Vet, Perú 11(2): 24-29.

4. Dávila A. 1996. Hidatidosis en la infancia. Contribución al tratamiento medicoquirúrgico. Revisión de los últimos 15 años en el INSN. Tesis de Médico Cirujano. Lima: Facultad de Medicina Humana, Univ Nacional Mayor de San Marcos. 78 p.

5. Jerray M, Benzarti M, Garrouche A, Klabi N, Hayouni A. 1992. Hydatid disease of the lungs: Study of 386 cases. Am Rev Resp Dis 146: 185-189.

6. Huamán M. 1999. Identificación de la cadena de transmisión de casos autóctonos de hidatidosis urbana. Tesis de Biólogo. Lima: Facultad de Ciencias Biológicas, Univ Nacional Mayor de San Marcos. 65 p.

7. Lozano D, López M, Caballero T. 2004. Hallazgos radiológicos en veinte casos de hidatidosis pulmonar en el Instituto Especializado de Salud del Niño, 2000-2002. Paediatrica 6(2): 81-86.

8. McManus D, Zhang W, Li J, Bartley P. 2003. Echinococcosis. Lancet 362 : 1295-1304.

9. Moro PL, McDonald J, Gilman R, Silva B, Verastegui M, Malqui $V$, Lescano G, et al. 1997. Epidemiology of Echinococcus granulosus infection in the central Peruvian Andes. B World Health Organ 75(6): 553-556.

10. Moro P, Lopera L, Cabrera M, Cabrera G, Silva B, Gilman R, Moro M. 2004. Short report: endemic focus of cystic echinococcosis in a coastal city of Peru. Am J Trop Med Hyg 71: 327329.

11. Muñoz P. 2007. Comentario editorial: Diagnóstico y tratamiento de la hidatidosis. Rev Chil Infectol 24: 153154.

12. Noemí I, Viovy A, Zamoran R, Blanco A, Revello D, Vojkovic M, Cerva J. 2003. Hidatidosis en la infancia: Albendazol en su tratamiento médico y quirúrgico. Rev Chil Infectol 20: 229-234.

13. Núñez E, Calero D, Estares L, Morales A. 2003. Prevalencia y factores de riesgo de hidatidosis en población general del distrito de Ninacaca-Pasco, Perú 2001. An Fac Med, Perú 64(1): 35-42.

14. Retamal C, Pérez C, Noemí I, Aguilera X, Apt W. 1994. Evaluación de las técnicas de doble difusión 5 e inmuelectroforesis en hidatidosis infantil en la casuística del decenio. Rev Chil Pediatr 65: 251-254.

15. Sapunar J. 1997. Hidatidosis. En: Parasitología médica. Atias A (ed). Chile: Ed. Mediterráneo. 339-354, 400-404 p.

16. Stiglich M, Vega-Briceño L, Gutierrez M, Trefogli P, Chiarella P. 2004. Hidatidosis pulmonar pediátrica: Reporte de 12 años de experiencia. Rev Chil Pediatr 75: 333-338.

17. Tamayo L, Pacheco R, Fernández $R$, Chungara J. 2004. Hidatidosis. Experiencia institucional. Rev Soc Bol Ped 43: 149-154. 